\title{
A Neglected Priority: Medical Doctors' Awareness of Travel Medicine in Turkey
}

\author{
İhmal Edilen Bir Öncelik: Türkiye'de Tıp Doktorlarnın Seyahat Tıbbı \\ Farkındalı̆ğ
}

(D) Varol Tunalı

Muğla University Faculty of Medicine, Department of Emergency, Muğla Turkey

\author{
Cite this article as: Tunalı V. A Neglected Priority: Medical Doctors' Awareness of Travel Medicine in Turkey.
}

Turkiye Parazitol Derg 2021;45(4):293-303.

\begin{abstract}
Objective: This study aimed to assess the attitudes and awareness of medical doctors practicing in Turkey about the discipline of travel medicine (TM).

Methods: This study was a cross-sectional survey conducted via the internet for 1 month between January 10, 2020 and February 10, 2020. The study participants were medical students or graduated medical doctors. The questionnaire used for the survey was broadly structured to measure participants' education on TM, perception of TM, and general knowledge about TM.

Results: A total of 502 participants were included in the study, of which 188 (37.4\%) were medical students and 314 (62.6\%) were medical doctors. Forty-nine out of $82(60 \%)$ medical faculties' students or graduates took part in this study. Experience in medicine was significantly associated with education and perception of TM but not with general knowledge about TM.

Conclusion: Our data indicate that medical doctors in Turkey are not familiar with the discipline (60\%) and that the medical doctors believe that the importance of TM is increasing (58.4\%). In total, $93.2 \%$ participants believe that social awareness about TM is insufficient. A total of $69 \%$ participants believe that infectious diseases make up the bulk of TM. To the best of our knowledge, this study is the first study to assess the awareness and attitudes of medical doctors in Turkey about TM.
\end{abstract}

Keywords: Travel medicine, medical education, migration medicine, Turkey

öz

Amaç: Bu çalışmanın amacı, Türkiye'de görev yapan tıp öğrencisi ve doktorların seyahat tıbbı (ST) disiplini hakkındaki tutum ve farkındalıklarını değerlendirmektir.

Yöntemler: Bu çalışma, 10 Ocak-10 Şubat 2020 tarihleri arasında internet üzerinden bir ay süreyle dağıtılan kesitsel bir anket çalışmasıdır. Katılımcılar tıp fakültesi öğrencileri ve mezun doktorlardan oluşmaktaydı. Anket, katılımcıların ST konusundaki; "A: ST Eğitimi; B: ST Algısı; C: ST Hakkında genel bilgi” parametrelerini ölçmek üzere yapılandırıldı.

Bulgular: Çalışmaya 502 katılımcı dahil edildi. Katılımcıların 188'i $(\% 37,4)$ tıp öğrencisi ve 314'ü $(\% 62,6)$ mezun tıp doktoru idi. Türkiye'deki 82 tıp fakültesinden 49'unun öğrenci ve mezunları (\%60) bu çalışmaya dahil edildi. Mesleki tecrübe, ST eğitimi ve algılanmasıyla önemli ölçüde ilişkili bulunurken, ST hakkında genel bilgi ile ilişkili olmadığı görüldü.

Sonuç: Verilerimiz, Türkiye'deki tıp doktorlarının ST disiplinine aşina olmamakla beraber (\%60), ST’nin öneminin arttığına inandıklarını $(\% 58,4)$ gösteriyor. Katılımcların \%93,2'si ST ile ilgili sosyal farkındalığın yetersiz olduğuna ve \%69'u ise bulaşıcı hastalıkların ST'nin en büyük bileşeni olduğuna inanıyor. Çalışmamız, bilindiği kadarıyla tıp doktorlarının ST konusundaki farkındalıklarını ve tutumlarını değerlendiren Türkiye'deki ve literatürdeki ilk çalışmadır.

Anahtar Kelimeler: Seyahat tıbbı, tıp eğitimi, göçmen sağlı̆̆ı, Türkiye

\section{INTRODUCTION}

Travel medicine (TM) defines all travel-related health and illness conditions. It is a medical discipline that can be dealt with in a wide range, from infectious diseases such as malaria, tourist diarrhea, yellow fever to problems encountered during travel such as trauma, embolism, jet-lag, sunburn, and all similar travel-related conditions $(1,2)$. Although TM is a comprehensive discipline, one of its main components is infectious diseases $(3,4)$. Among these infectious diseases, parasitic and tropical diseases are the major causes of mortality and morbidity (5-7).

International travel is one of the most important routes for the spread of infectious diseases (8). It is reported that in 2018, 1.4 billion people traveled internationally and the world tourism volume 
exceeded $\$ 1.7$ trillion (9). TM, which is a relatively new field of medicine, is a discipline that directly affects more than 1.4 billion people all over the world and indirectly concerns the whole world population. TM is expected to develop rapidly due to reasons such as increased travel opportunities and facilitation of international travel in general (10). There are travel disease clinics and centers in Europe, Australia, and North America, and collaborations such as GeoSentinel and EuroTravNet try to provide worldwide surveillance $(3,4)$.

Turkey is situated as a geographical bridge between three continents; Asia, Africa, and Europe. Owing to its geographical location, it is a major route for displaced people like migrants, asylum seekers, and refugees to get to Europe. According to the United Nations Refugee Agency (UNHCR), 57\% of UNHCR refugees came from 3 countries; Syria, Afghanistan and South Sudan (11). Turkey is the main route for Syrian and Afghan refugees to get in Europe and thus Turkey is the World's largest refugee-hosting country with more than 3.7 million refugees (11). There have been several reports of imported cases of malaria and leishmaniasis, both among refugees and Turkish residents $(12,13)$. In addition to that, with its temperate climate, long coastal line, and rich cultural heritage, Turkey is an important destination for international tourists. According to the World Tourism Organization's latest report, Turkey is the $6^{\text {th }}$ top destination for tourist arrivals worldwide, receiving more than 46 million tourists in 2018 (9).

This study aims to assess the awareness and attitudes of medical students and medical doctors practicing in Turkey, about the discipline of TM.

\section{METHODS}

\section{Design}

This study was a cross-sectional survey that was distributed via the internet for one month between 10 January and 10 February 2020. The participant's consent was obtained by the acceptance for the completion of the questionnaire and all the participant's e-mail addresses were demanded for the authentication via e-mail. All the participants were informed that the answers would be published anonymously.

\section{Subjects}

The targeted participants were either medical students or graduated medical doctors over 18 years of age. Medical students were grouped as; $1^{\text {st }}-3^{\text {rd }}$ grade and $4^{\text {th }}-6^{\text {th }}$ grade medical students and medical doctors as; to 10 years after graduation, 10 to 20 years after graduation and 20 years or more after graduation.

\section{Questionnaire}

The questionnaire was pretested for the validity of questions by a group of ten physicians with clinical experience in the field of TM. This group of physicians consists of infectious diseases, internal medicine, emergency medicine, clinical microbiology and clinical parasitology specialists working in various clinics around Turkey. The questionnaire was broadly structured in three objective categories to measure participants'; A. Education on TM, B. Perception of TM, C. General knowledge about TM. The statements in the questionnaire were randomly placed and participants were asked to answer the statements from a five-point likert scale. The participants were asked to give points to each statement, from 1 to 5 where the numbers stand for; 1 . Strongly disagree - 5 .
Strongly agree. The statements in the questionnaire are shown in Table 1.

\section{Statistical Analysis}

Descriptive data analysis of means was conducted using the Statistics package (SPSS Statistics 22 version). Multinomial logistic regression was used with the reference category "medical student $1^{\text {st }}-3^{\text {rd }}$ grade". Results were depicted with B coefficients, and $95 \%$ confidence intervals. Statistical analysis of each objective category for every participant group has been conducted. Five participant groups have been compared based on the experience level (years passed in medical practice starting from the $1^{\text {st }}$ year of medical faculty).

\section{RESULTS}

There were 502 participants included in the study. The participant's answers to statements between Statement-1 (S-1) and $\mathrm{S}-15$ from a scale of 1 to 5 respectively are provided in Table 1. Of all participants $(n=502), 22.7 \%$ were medical students in the $1^{\text {st }}-3^{\text {rd }}$ grade and $14.7 \%$ were $4^{\text {th }}-6^{\text {th }}$ grade. Participants who are medical doctors who have less than 10 years of experience, 10-20 years of experience and more than 20 years of experience make up $35.9 \%, 9 \%$ and $17.7 \%$ of all participants respectively (Figure 1 ).

Fourty-nine out of 82 (60\%) Turkish medical faculties' students or graduates have taken part in this study. These medical faculties are distributed across all geographical regions of Turkey.

The average points given to every individual statement by participant groups have been compared statistically. Mean value has been calculated for each objective category (A, B, C) based on the relevant statements. The mean value for each objective category is presented for each participant group in Figure 2.

Statistical analysis of each objective category for each participant group based on their experience levels have been calculated (Table 2). Experience in medicine was significantly associated with education on TM $\left(\mathrm{x}^{2}: 12.8\right.$, df: $\left.4, \mathrm{p}=0.01\right)$, and perception of TM ( $x^{2}: 13.0$, df: 4, $\left.\mathrm{p}=0.01\right)$. The association between experience in medicine and general knowledge about TM was below the significance level ( $\mathrm{x}^{2}: 9.06$, df: $\left.4, \mathrm{p}=0.06\right)$. Detailed statistical analysis reports for each statement and objective category has been provided as Supplementary material.

\section{DISCUSSION}

Travel history is a very important component of anamnesis. Every physician, regardless of their specializations, institutes or positions, should include travel history for every anamnesis they take (10). A thorough travel history will enable the physician to better identify the possible causes of the illness and enable him/ her to contain it in cases of infectious diseases (14). The current Coronavirus disease-2019 (COVID-19) pandemic displays the fragility of our global health administration mechanisms against novel or re-emerging infectious agents (15). Health professionals and, medical doctors in particular, are the main actors for the prevention and management of epidemics or even pandemics caused by these agents. Therefore, their awareness and knowledge about TM is crucial to prevent and contain these situations.

Interpretation of our data indicates that most of the medical doctors in Turkey have not received any education about TM (84.4\% of participants) but they are aware of the need for 


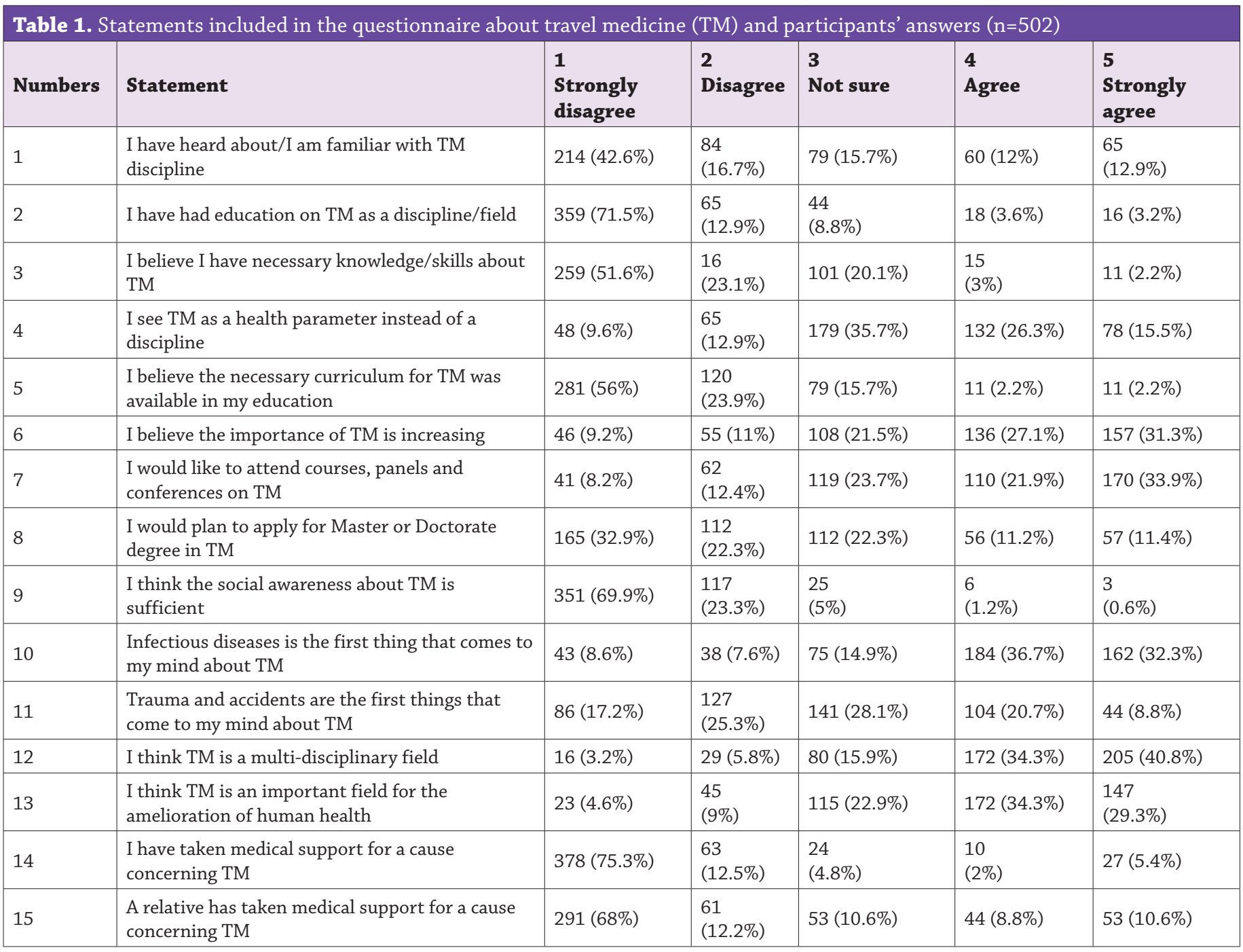

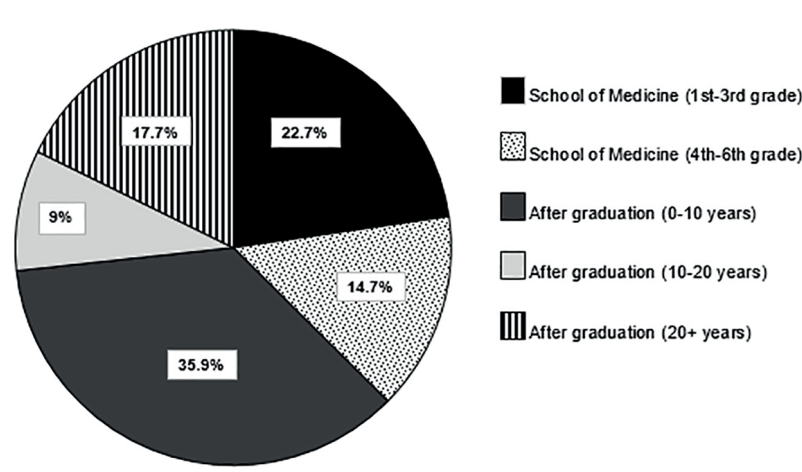

Figure 1. The distribution of participants according to experience groups

education possibilities about it. Perception of TM (Category B) was the highest-ranking objective category for all the study groups, while education on TM (Category A) was the lowest, pointing out the awareness of medical doctors about TM (Figure 2). Among all study groups, $70 \%$ of the participants believe that the necessary curriculum for TM was not available to them. Our data also shows that even if most of them are not familiar with the discipline of TM (60\%), most of them believe the importance of TM is increasing (58.4\%) and they are willing to participate in

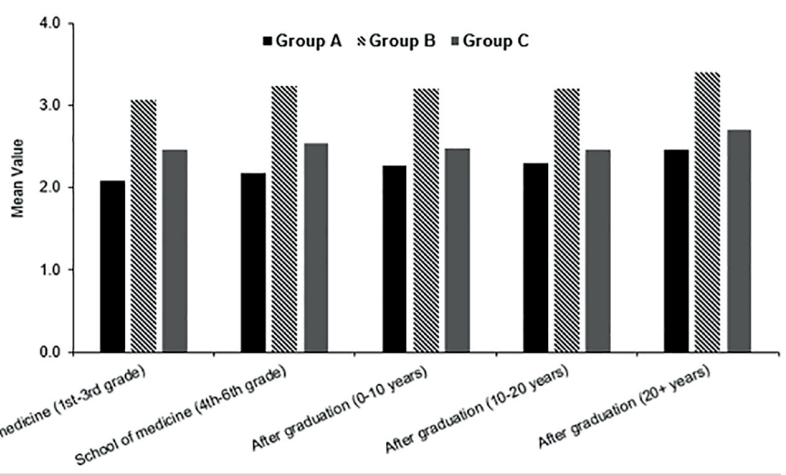

Figure 2. Mean values of the $A, B$ and $C$ objective groups

educational works about TM (55.8\%). 93.2\% of the participants believe the social awareness about TM is insufficient which in our opinion points out to the fact that if physicians' awareness is improved, social awareness about TM will improve proportionally. Most of the physicians in Turkey believe, infectious diseases make up the bulk of TM (69\%) and TM is important for the amelioration of human health (63.6\%).

Our study indicates that TM education should be included in the curriculum of medical faculties in Turkey. Several studies have been conducted about the importance of TM education in medical faculties. A single question travel history is no longer sufficient 


\begin{tabular}{|c|c|c|c|c|c|}
\hline & $\begin{array}{l}\text { Medical faculty } \\
1^{\text {st }}-3^{\text {rd }} \text { grades }\end{array}$ & $\begin{array}{l}\text { Medical faculty } 4^{\text {th }} \text { - } \\
6^{\text {th }} \text { grades }\end{array}$ & $\begin{array}{l}\text { Medical doctors } \leq 10 \\
\text { years exp. }\end{array}$ & $\begin{array}{l}\text { Medical doctors } 10- \\
20 \text { years exp. }\end{array}$ & $\begin{array}{l}\text { Medical } \\
\text { doctors } \geq 20 \\
\text { years exp. }\end{array}$ \\
\hline & $\begin{array}{l}\text { Mean B } \\
(\mathrm{SD}) \quad(95 \% \mathrm{CI})\end{array}$ & $\begin{array}{l}\text { Mean B } \\
(\mathrm{SD}) \quad(95 \% \mathrm{CI})\end{array}$ & $\begin{array}{l}\text { Mean B } \\
(\mathrm{SD}) \quad(95 \% \mathrm{CI})\end{array}$ & $\begin{array}{l}\text { Mean B } \\
(\mathrm{SD}) \quad(95 \% \mathrm{CI})\end{array}$ & $\begin{array}{l}\text { Mean B } \\
(\mathrm{SD}) \quad(95 \% \mathrm{CI})\end{array}$ \\
\hline $\begin{array}{l}\text { Education on TM } \\
\text { (category A) }\end{array}$ & $\begin{array}{l}12.530 \text { (ref) } \\
(3.73)\end{array}$ & $\begin{array}{ll}13.05 & 0.02 \\
(4.59) & (-0.39-0.92)\end{array}$ & $\begin{array}{l}13.60 .05 \\
(5.12)(-0.00-0.10)\end{array}$ & $\begin{array}{l}13.80 .60 \\
(4.88)(-0.01-0.13)\end{array}$ & $\begin{array}{l}14.860 .10^{* *} \\
(5.11)(0.04- \\
0.16)\end{array}$ \\
\hline $\begin{array}{l}\text { Perception of TM } \\
\text { (category B) }\end{array}$ & $\begin{array}{l}17.710 \text { (ref) } \\
(3.65)\end{array}$ & $\begin{array}{ll}18.51 & 0.05 \\
(3.72) & (-0.02-0.13)\end{array}$ & $\begin{array}{ll}18.45 & 0.04 \\
(3.93) & (-0.01-0.11)\end{array}$ & $\begin{array}{ll}18.73 & 0.06 \\
(3.85) & (-0.02-0.16)\end{array}$ & $\begin{array}{l}19.620 .13^{* *} \\
(3.76)(0.05- \\
0.21)\end{array}$ \\
\hline $\begin{array}{l}\text { General knowledge about } \\
\text { TM (category C) }\end{array}$ & $\begin{array}{l}9.790 \text { (ref) } \\
(2.88)\end{array}$ & $\begin{array}{ll}10.13 & 0.04 \\
(2.60) & (-0.06-0.15)\end{array}$ & $\begin{array}{l}9.880 .01 \\
(2.57)\left(-0.07-{ }^{*} 0.10\right)\end{array}$ & $\begin{array}{l}9.860 .01 \\
(1.90)(-0.12-0.14)\end{array}$ & $\begin{array}{l}10.840 .13^{*} \\
(3.16)(0.03- \\
0.23)\end{array}$ \\
\hline
\end{tabular}

for coping with travel-related medical conditions and especially for the emerging and re-emerging diseases (16). A recent study by Flaherty et al. (17) discusses the benefits of integrating special study modules to undergraduate medical curriculum. In addition to that, general practitioners' awareness and knowledge about TM should be constantly increased and kept up-to-date (18).

\section{CONCLUSION}

Data of this study was collected in a unique interval between January-February 2020, just weeks before World Health Organization declared COVID-19 as a pandemic. Our study outlines the need for TM education both for undergraduate and graduate medical doctors. There is significant difference between undergraduate and graduate medical doctors for the awareness or attitudes about TM which points out to the fact that medical doctors' awareness of TM is increasing with work experience. Considering Turkey's geopolitical position, tourist potential, İstanbul's possible role as an important hub in terms of airline traffic, and the ongoing global refugee crisis, TM clinics are considered to be a necessity for Turkey.

Our study is the first study to assess the awareness and attitudes of medical doctors about TM in Turkey and to the best of our knowledge in the literature.

\section{ACKNOWLEDGEMENTS}

The authors would like to thank Dr. Sila Selin Tunalı for her invaluable help in distributing the questionnaire nationwide and Dr. Umut Kirl for assessing the data. We also would like to thank Turkish Society of Microbiology Study Group for Infections Related to Migration and Travel for their support.

\section{* Ethics}

Ethics Committee Approval: This study was a cross-sectional survey that was distributed via the internet for one month between 10 January and 10 February 2020.

Informed Consent: Informed consent was obtained.

Peer-review: Internally peer-reviewed.

Financial Disclosure: The author declared that this study received no financial support.

\section{REFERENCES}

1. Steffen R, DuPont HL. Travel Medicine: What's That? J Travel Med 1994; 1: 1-3.

2. Aw B, Boraston S, Botten D, Cherniwchan D, Fazal H, Kelton T, et al. Travel medicine: what's involved? When to refer? Can Fam Physician 2014; 60: 1091-103.

3. Schlagenhauf P, Weld L, Goorhuis A, Gautret P, Weber R, von Sonnenburg F, et al. Travel-associated infection presenting in Europe (2008-12): an analysis of EuroTravNet longitudinal, surveillance data, and evaluation of the effect of the pre-travel consultation. Lancet Infect Dis 2015; 15: 55-64.

4. Leder K, Torresi J, Libman MD, Cramer JP, Castelli F, Schlagenhauf P, et al. GeoSentinel surveillance of illness in returned travelers, 2007-2011. Ann Intern Med 2013; 158: 456-68.

5. Showler AJ, Wilson ME, Kain KC, Boggild AK. Parasitic diseases in travelers: a focus on therapy. Expert Rev Anti Infect Ther 2014; 12: 497521.

6. Norman FF, Monge-Maillo B, Martínez-Pérez Á, Perez-Molina JA, LópezVélez R. Parasitic infections in travelers and immigrants: part I protozoa. Future Microbiol 2015; 10: 69-86.

7. Norman FF, Monge-Maillo B, Martínez-Pérez Á, Perez-Molina JA, LópezVélez R. Parasitic infections in travelers and immigrants: part II helminths and ectoparasites. Future Microbiol 2015; 10: 87-99.

8. Baker KS, Dallman TJ, Ashton PM, Day M, Hughes G, Crook PD, et al. Intercontinental dissemination of azithromycin-resistant shigellosis through sexual transmission: a cross-sectional study. Lancet Infect Dis 2015; 15: 913-21.

9. (2019) International Tourism Highlights, 2019 Edition. World Tourism Organization (UNWTO)

10. Tunalı V, Turgay N. The Concept of Travel Medicine and the Actual Situation of Travel-Related Illnesses. Turkiye Parazitol Derg 2017; 41: 114-8.

11. UNHCR - Figures at a Glance. https://www.unhcr.org/figures-at-a-glance. html. Accessed 16 Mar 2020.

12. Tünger Ö, Çakmak A, Özbilgin A, Tunalı V, Çetin ÇB. Imported Malaria in Turkey: The Importance of Diagnosis and Treatment of Plasmodium falciparum/Plasmodium vivax Mixed Infection. Turkiye Parazitol Derg 2018; 42: 164-7.

13. Özbilgin A, Gencoglan G, Tunali V, Çavuş İ, Yıldırım A, Gündüz C, Harman M. Refugees at the Crossroads of Continents: A Molecular Approach for Cutaneous Leishmaniasis Among Refugees in Turkey. Acta Parasitol 2020; 65: 136-43.

14. Rodriguez-Morales AJ, Schlagenhauf P. Zoonoses and travel medicine: "one world--one health". Travel Med Infect Dis 2014; 12: 555-6. 
15. Peeri NC, Shrestha N, Rahman MS, Zaki R, Tan Z, Bibi S, et al. The SARS, MERS and novel coronavirus (COVID-19) epidemics, the newest and biggest global health threats: what lessons have we learned? Int $\mathrm{J}$ Epidemiol 2020; 49: 717-26.

16. Kozarsky PE, Steffen R. Travel medicine education-what are the needs? J Travel Med 2016; 23.

\section{Supplementary Material}

\begin{tabular}{r|rrrrr} 
Variable & Obs & Mean & Std. Dev. & Min & Max \\
\hline educat & 114 & 12.53509 & 3.73024 & 6 & 25
\end{tabular}

- summarize educat if var00001==1

\begin{tabular}{c|ccccr} 
Variable & Obs & Mean & Std. Dev. & Min & Max \\
educat & 74 & 13.05405 & 4.595686 & 6 & 30
\end{tabular}

. summarize educat if $\operatorname{var} 00001=2$

\begin{tabular}{r|ccccr} 
Variable & Obs & Mean & Std. Dev. & Min & Max \\
\hline educat & 180 & 13.6 & 5.12154 & 6 & 29
\end{tabular}

. summarize educat if var00001== 3

\begin{tabular}{r|ccccr} 
Variable & Obs & Mean & Std. Dev. & Min & Max \\
\hline educat & 45 & 13.8 & 4.887833 & 6 & 25
\end{tabular}

. summarize educat if var00001==4

\begin{tabular}{r|rrrrr} 
Variable & Obs & Mean & Std. Dev. & Min & Max \\
\hline educat & 89 & 14.86517 & 5.110575 & 6 & 30
\end{tabular}

Experience VS. education on TM . summarize educat if $\operatorname{var} 00001==0$

\begin{tabular}{|c|c|c|c|c|c|c|}
\hline var00001 & Coef. & Std. Err. & $\mathrm{z}$ & $P>|z|$ & [958 Conf. & Interval] \\
\hline tip_1_3_donem & \multicolumn{6}{|c|}{ (base outcome) } \\
\hline \multicolumn{7}{|l|}{ tip_4_6_donem } \\
\hline educat & .0266785 & .0337284 & 0.79 & 0.429 & -.0394279 & .0927849 \\
\hline _cons & -.7733529 & .4580546 & -1.69 & 0.091 & -1.671123 & .1244175 \\
\hline \multicolumn{7}{|l|}{ md_0_10 } \\
\hline educat & .0520413 & .0270363 & 1.92 & 0.054 & -.000949 & . 1050315 \\
\hline _cons & -.2223583 & .3689924 & -0.60 & 0.547 & -.9455701 & .5008534 \\
\hline \multicolumn{7}{|l|}{ md_10_20 } \\
\hline educat & .0607457 & .0379471 & 1.60 & 0.109 & -.0136292 & .1351206 \\
\hline -cons & -1.727898 & .537732 & -3.21 & 0.001 & -2.781834 & -.6739628 \\
\hline \multicolumn{7}{|l|}{ md_20_ustu } \\
\hline educat & .1029062 & .0304421 & 3.38 & 0.001 & .0432408 & .1625717 \\
\hline _cons & -1.649625 & .4394342 & -3.75 & 0.000 & -2.5109 & -.7883501 \\
\hline
\end{tabular}

LR chi2(4) $=12.80$

Prob > chi2 $=0.0123$

Experience in medicine was significantly associated with education on TM $\left(\mathrm{x}^{2}: 12.8\right.$, df:4, $\mathrm{p}=0.01$ )
17. Flaherty G, Thong Zi Yi C, Browne R. The missing link: introducing travel medicine into the undergraduate medical curriculum. J Travel Med 2016; 23.

18. Leder K, Bouchaud O, Chen LH. Training in Travel Medicine and General Practitioners: A Long-Haul Journey! J Travel Med 2015; 22: 357-60.

\begin{tabular}{|c|c|c|c|c|c|}
\hline Variable & obs & Mean & Std. Dev. & Min & Max \\
\hline percept & 114 & 17.71053 & 3.654852 & 6 & 25 \\
\hline Variable & obs & Mean & Std. Dev. & Min & Max \\
\hline percept & 74 & 18.51351 & 3.724202 & 10 & 28 \\
\hline Variable & obs & Mean & Std. Dev. & $\operatorname{Min}$ & Max \\
\hline percept & 180 & 18.45 & 3.93562 & 6 & 28 \\
\hline - summarize & if vo & $001==3$ & & & \\
\hline variable & obs & Mean & Std. Dev. & $\operatorname{Min}$ & Max \\
\hline percept & 45 & 18.73333 & 3.857696 & 8 & 25 \\
\hline . summarize & if va & $001==4$ & & & \\
\hline Variable & obs & Mean & Std. Dev. & Min & Max \\
\hline percept & 89 & 19.62921 & 3.76401 & 9 & 30 \\
\hline
\end{tabular}

Experience VS. perception on TM

summarize percept if var00001 $==0$

\begin{tabular}{|c|c|c|c|c|c|c|}
\hline var00001 & Coef. & Std. Err. & $z$ & $P>|z|$ & [958 Conf. & Interval] \\
\hline tip_1_3_donem & \multicolumn{6}{|c|}{ (base outcome) } \\
\hline \multicolumn{7}{|l|}{ tip_4_6_donem } \\
\hline percept & .0544165 & .0391926 & 1.39 & 0.165 & -.0223996 & .1312325 \\
\hline _cons & -1.417985 & .7292983 & -1.94 & 0.052 & -2.847383 & .0114137 \\
\hline \multicolumn{7}{|l|}{ md_0_10 } \\
\hline percept & .049974 & .0312045 & 1.60 & 0.109 & -.0111857 & .1111338 \\
\hline _cons & -.4469888 & .5747053 & -0.78 & 0.437 & -1.573391 & .6794129 \\
\hline \multicolumn{7}{|l|}{ md_10_20 } \\
\hline percept & .0699616 & .0467172 & 1.50 & 0.134 & -.0216025 & .1615257 \\
\hline _cons & -2.204895 & .8803895 & -2.50 & 0.012 & -3.930427 & -.4793634 \\
\hline \multicolumn{7}{|l|}{ md_20_ustu } \\
\hline percept & .1356885 & .0388088 & 3.50 & 0.000 & .0596246 & .2117523 \\
\hline _cons & -2.783905 & .7457051 & -3.73 & 0.000 & -4.24546 & -1.32235 \\
\hline
\end{tabular}

LR chi2(4) $=13.01$

Prob > chi2 $=0.0112$

Experience in medicine was significantly associated with perception on TM $\left(x^{2}: 13.0, d f: 4, p=0.01\right)$ 
Experience vs. general knowledge about $\mathrm{TM}^{* *}$

. summarize generall if var00001==0

\begin{tabular}{c|ccccc} 
Variable & Obs & Mean & Std. Dev. & Min & Max \\
\hline generall & 114 & 9.798246 & 2.881678 & 4 & 19
\end{tabular}

- summarize generall if var00001== 1

\begin{tabular}{c|ccccc} 
Variable & Obs & Mean & Std. Dev. & Min & Max \\
generall & 74 & 10.13514 & 2.603077 & 6 & 18
\end{tabular}

- summarize generall if var00001== 2

\begin{tabular}{c|ccccr} 
Variable & Obs & Mean & Std. Dev. & Min & Max \\
\hline generall & 180 & 9.888889 & 2.578139 & 4 & 19
\end{tabular}

. summarize generall if var00001== 3

\begin{tabular}{c|ccccc} 
Variable & Obs & Mean & Std. Dev. & Min & Max \\
\hline generall & 45 & 9.866667 & 1.902152 & 7 & 16
\end{tabular}

. sumarize generall if var00001== 4

\begin{tabular}{c|ccccc} 
Variable & obs & Mean & Std. Dev. & Min & Max \\
\hline generall & 89 & 10.8427 & 3.165506 & 4 & 20
\end{tabular}

\begin{tabular}{|c|c|c|c|c|c|c|}
\hline $\operatorname{var} 00001$ & Coef. & Std. Err. & $\mathrm{z}$ & $P>|z|$ & [958 Conf. & Interval] \\
\hline tip_1_3_donem & \multicolumn{6}{|c|}{ (base outcome) } \\
\hline \multicolumn{7}{|l|}{ tip_4_6_donem } \\
\hline generall & .0475996 & .0559659 & 0.85 & 0.395 & -.0620915 & .1572908 \\
\hline _cons & -.9064257 & .5790429 & -1.57 & 0.117 & -2.041329 & .2284775 \\
\hline \multicolumn{7}{|l|}{ md_0_10 } \\
\hline generall & .0132252 & .0457928 & 0.29 & 0.773 & -.076527 & .1029775 \\
\hline _cons & .3265773 & .465918 & 0.70 & 0.483 & -.5866052 & 1.23976 \\
\hline \multicolumn{7}{|l|}{ md_10_20 } \\
\hline generall & .010012 & .0672181 & 0.15 & 0.882 & -.121733 & .1417571 \\
\hline _cons & -1.027978 & .684916 & -1.50 & 0.133 & -2.370389 & .3144329 \\
\hline \multicolumn{7}{|l|}{ md_20_ustu } \\
\hline generall & .1351074 & .0515333 & 2.62 & 0.009 & .034104 & .2361109 \\
\hline _cons & -1.638807 & .5507261 & -2.98 & 0.003 & -2.718211 & -.5594038 \\
\hline
\end{tabular}

LR chi2 $(4)=9.06$

Prob > chi2 $=0.0597$

The association between experience in medicine and general knowledge about TM was below the significance level $\left(x^{2}: 9.06, \mathrm{df}: 4, \mathrm{p}=0.06\right)$

\begin{tabular}{r|rrrrr} 
Variable & Obs & Mean & Std. Dev. & Min & Max \\
\hline sl & 114 & 1.789474 & 1.132572 & 1 & 5
\end{tabular}

summarize sl if var00001== 1

\begin{tabular}{r|rrrrr} 
Variable & Obs & Mean & Std. Dev. & Min & Max \\
\hline sl & 74 & 2.216216 & 1.416829 & 1 & 5
\end{tabular}

. summarize s1 if $\operatorname{var} 00001==2$

\begin{tabular}{r|rrrrr} 
Variable & Obs & Mean & Std. Dev. & Min & Max \\
\hline sl & 180 & 2.427778 & 1.398445 & 1 & 5
\end{tabular}

. summarize s1 if $\operatorname{var} 00001==3$

\begin{tabular}{r|rrrrr} 
Variable & Obs & Mean & Std. Dev & Min & Max \\
\hline sl & 45 & 2.555556 & 1.485621 & 1 & 5
\end{tabular}

- summarize $s 1$ if $\operatorname{var} 00001==4$

\begin{tabular}{r|rrrrr} 
Variable & Obs & Mean & Std. Dev & Min & Max \\
\hline s1 & 89 & 3.011236 & 1.654844 & 1 & 5
\end{tabular}

** experience vs. $s 1^{* *}$

summarize $\mathrm{s} 1$ if $\operatorname{var} 00001==0$

\begin{tabular}{|c|c|c|c|c|c|c|c|}
\hline & vas000001 & coos. & sta. Err. & $=$ & $p>1 z 1$ & cost conf. & Interososi] \\
\hline & $\left.: 1 p_{-} 1_{-}\right\}_{-}$donen & (base outcerer & & & & & \\
\hline & $\begin{array}{r}\text { EtP_4_6_donen } \\
\text { on } \\
\text {-cons }\end{array}$ & $\begin{array}{r}.2621657 \\
\therefore .9538955\end{array}$ & $\begin{array}{l}.1170727 \\
.2798551\end{array}$ & $\begin{array}{r}2.24 \\
-3.41\end{array}$ & $\begin{array}{l}0.025 \\
0.001\end{array}$ & $\begin{array}{r}.0327074 \\
-1.502401\end{array}$ & $\begin{array}{r}.991624 \\
-4053896\end{array}$ \\
\hline $\mathrm{na}_{0} \mathrm{O}_{-10}$ & $\begin{array}{r}51 \\
\text { _cons }\end{array}$ & $\begin{array}{l}.36715294 \\
-.306502515\end{array}$ & $\begin{array}{l}.0967957 \\
.2271463\end{array}$ & $\begin{array}{r}3.79 \\
-1.36\end{array}$ & $\begin{array}{l}0.000 \\
0.174\end{array}$ & $\begin{array}{r}.1775247 \\
-.7538\end{array}$ & $\begin{array}{l}.9567902 \\
.1355972\end{array}$ \\
\hline nd ${ }_{-10} 0_{-20}$ & $\begin{array}{r}\Delta 1 \\
\text { - cons }\end{array}$ & $\begin{array}{r}.4266247 \\
-1.842557\end{array}$ & $\begin{array}{c}.1299885 \\
.394073\end{array}$ & $\begin{array}{r}3.28 \\
-5.34\end{array}$ & $\begin{array}{l}0.001 \\
0.000\end{array}$ & $\begin{array}{l}.1716518 \\
-2.518887\end{array}$ & $\begin{array}{r}-6811975 \\
-1.1166226\end{array}$ \\
\hline nd_ze_ustu & $\begin{array}{r}s 1 \\
\text { _cone }\end{array}$ & $\begin{array}{r}.6258705 \\
-1.725306\end{array}$ & $\begin{array}{l}.1090565 \\
.2960162\end{array}$ & $\begin{array}{r}5.74 \\
-5.79\end{array}$ & $\begin{array}{l}0.000 \\
0.000\end{array}$ & $\begin{array}{r}.4121241 \\
-2.2951897\end{array}$ & $\begin{array}{r}-40396169 \\
-1.1135125\end{array}$ \\
\hline
\end{tabular}

\begin{tabular}{r|ccccc} 
Variable & obs & Mean & Std. Dev. & Min & Max \\
\hline${ }_{52}$ & ${ }^{114}$ & 1.192982 & .5363223 & 1 & 5
\end{tabular}

\begin{tabular}{r|rrrrr} 
Variable & obs & Mean & Std. Dev. & Min & Max \\
\hline S2 & 74 & 1.608108 & .9625532 & 1 & 5
\end{tabular}

. sumnarize s2 if varovo01= $=2$

\begin{tabular}{c|ccccc} 
Variable & obs & Mean & Std. Dev. & Min & Max \\
\hline-2 & 180 & 1.705556 & 1.09696 & 1 & 5
\end{tabular}

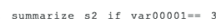

\begin{tabular}{|c|c|c|c|c|c|}
\hline variable & obs & mean & sta. pev. & $\operatorname{Min}$ & \\
\hline 32 & 45 & 1.6 & .96295 & 1 & \\
\hline . sunnarize $s 2$ if varo00 & & & & & \\
\hline variable & $\mathrm{obs}$ & Mean & std. Dev. & $\min$ & $\max$ \\
\hline $\mathrm{s} 2$ & 89 & 595506 & 1.212802 & 1 & \\
\hline
\end{tabular}




\begin{tabular}{|c|c|c|c|c|c|c|}
\hline varo00001 & RRR & sta. Brr. & $=$ & $p>|z|$ & 1958 cont. & Interval] \\
\hline 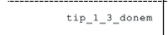 & (base outcone) & & & & & \\
\hline 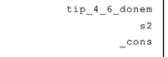 & $\begin{array}{l}1.936276 \\
.2668848\end{array}$ & $\begin{array}{l}.3962195 \\
.082228281\end{array}$ & $\begin{array}{r}3.23 \\
-4.28\end{array}$ & $\begin{array}{l}0.001 \\
0.000\end{array}$ & $\begin{array}{l}1.296541 \\
.144151508\end{array}$ & $\begin{array}{l}2.891668 \\
.4867374\end{array}$ \\
\hline nd ${ }_{-}{ }_{-}{ }^{10}$ & $\begin{array}{r}2.09888 \\
.56378797\end{array}$ & $\begin{array}{l}.38910989 \\
.219844949\end{array}$ & $\begin{array}{l}4.00 \\
-2.216\end{array}$ & $\begin{array}{l}0.000 \\
0.031\end{array}$ & $\begin{array}{l}1.459833 \\
.3352833\end{array}$ & $\begin{array}{l}3.018502 \\
.9479962\end{array}$ \\
\hline nd $\_10 \_20$ & $\begin{array}{r}1.922226 \\
-1.62991\end{array}$ & $\begin{array}{l}.42935999 \\
.055768767\end{array}$ & $\begin{array}{r}2.93 \\
-5.13\end{array}$ & $\begin{array}{l}0.003 \\
0.0000\end{array}$ & $\begin{array}{l}1.2401272 \\
.08614341\end{array}$ & $\begin{array}{l}2.978054 \\
3.3264146\end{array}$ \\
\hline na_ze_ustu & $\begin{array}{l}. .9124398 \\
.3244285\end{array}$ & $\begin{array}{l}.38299798 \\
.096732424\end{array}$ & $\begin{array}{c}3.25 \\
-3.78 \\
-128\end{array}$ & $\begin{array}{l}0.001 \\
0.000\end{array}$ & $\begin{array}{l}1.283944 \\
.18005518\end{array}$ & $\begin{array}{r}2.83346 \\
.58198997\end{array}$ \\
\hline
\end{tabular}

** experience vs $\mathrm{s} 2 * *$

. summarize $\mathrm{s} 2$ if $\operatorname{var} 00001==0$

\begin{tabular}{|c|c|c|c|c|c|c|}
\hline varoooop 1 & coos. & sta. krr. & $=$ & $00 \mid 21$ & I958 conf. & Intervas 1] \\
\hline 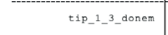 & (bese outcore) & & & & & \\
\hline $\begin{array}{r}\text { Eip_-46__donem } \\
\text { s3 } \\
- \text { cons }\end{array}$ & $\begin{array}{l}.2505956 \\
-.8345066\end{array}$ & $\begin{array}{l}.1727912 \\
.31707966\end{array}$ & $\begin{array}{r}1.45 \\
-2.63\end{array}$ & $\begin{array}{l}0.147 \\
0.008\end{array}$ & $\begin{array}{r}-.08961 \\
-1.455971 \\
-1.45\end{array}$ & $\begin{array}{r}.5891322 \\
-.2130421 \\
\end{array}$ \\
\hline ma__o_-10 & 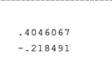 & $\begin{array}{l}.1003735 \\
.2569992\end{array}$ & $\begin{array}{r}2.88 \\
-0.85\end{array}$ & $\begin{array}{l}0.004 \\
0.395\end{array}$ & $\begin{array}{r}.12069797 \\
-.72210202\end{array}$ & $\begin{array}{l}.679733 \\
.285198 \\
.258\end{array}$ \\
\hline$\overline{a a_{-}-1020}$ & $\begin{array}{l}.3352281 \\
-1.479916\end{array}$ & $\begin{array}{l}.1945455 \\
.3729359\end{array}$ & $\begin{array}{r}1.72 \\
-3.97\end{array}$ & $\begin{array}{l}0.005 \\
0.000\end{array}$ & $\begin{array}{l}-.0466797 \\
-2,21210101\end{array}$ & $\begin{array}{r}.717: 39 \\
-.7482194\end{array}$ \\
\hline md_20__uetu & $\begin{array}{l}.15733555 \\
-1.54272701\end{array}$ & $\begin{array}{l}.1538281 \\
.315744\end{array}$ & $\begin{array}{r}4.92 \\
-5.20 \\
-520\end{array}$ & $\begin{array}{l}0.000 \\
0.000\end{array}$ & $\begin{array}{r}-45858391 \\
-2,2261554\end{array}$ & $\begin{array}{r}1.058833 \\
-1.023849\end{array}$ \\
\hline
\end{tabular}

\begin{tabular}{|c|c|c|c|c|c|c|}
\hline va 000001 & RRR & std. $8 r x$. & $=$ & $p>\mid 21$ & lost conf. & Intervall \\
\hline 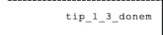 & (base outcona) & & & & & \\
\hline 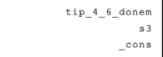 & $\begin{array}{l}.2 .289778 \\
.9340886\end{array}$ & $\begin{array}{l}.22192909 \\
.1376006\end{array}$ & $\begin{array}{r}1.45 \\
-2.63\end{array}$ & $\begin{array}{l}0.147 \\
0.008\end{array}$ & $\begin{array}{l}.9157966 \\
.2331738\end{array}$ & $\begin{array}{l}1.80242929 \\
.8081221\end{array}$ \\
\hline $\begin{array}{r}83 \\
\text { sones } \\
\text { cone }\end{array}$ & $\begin{array}{l}.4998713 \\
.8037300\end{array}$ & $\begin{array}{l}.2007969 \\
.2065179\end{array}$ & $\begin{array}{r}2.88 \\
-0.85\end{array}$ & $\begin{array}{l}0.009 \\
0.395\end{array}$ & $\begin{array}{l}1.138236 \\
.48557302\end{array}$ & $\begin{array}{l}1.97335252 \\
1.339922\end{array}$ \\
\hline $\begin{array}{r}50 \\
-\cos \\
-\cos \end{array}$ & $\begin{array}{l}1.398259 \\
.22782829\end{array}$ & $\begin{array}{l}.27245717 \\
.0898656\end{array}$ & $\begin{array}{c}1.72 \\
-3.97\end{array}$ & $\begin{array}{l}0.085 \\
0.000\end{array}$ & $\begin{array}{l}.95493931 \\
.1098985\end{array}$ & $\begin{array}{l}2.049558 \\
.4732084\end{array}$ \\
\hline nd_20_ustu & 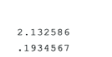 & $\begin{array}{l}.32850516 \\
.0601038\end{array}$ & $\begin{array}{c}4.92 \\
-5.20\end{array}$ & $\begin{array}{l}0.000 \\
0.0000\end{array}$ & $\begin{array}{l}1.577795 \\
.10401894\end{array}$ & $\begin{array}{l}2.883005 \\
.3592098\end{array}$ \\
\hline
\end{tabular}

*** experience $s 4^{* *}$

summarize s4 if var00001 $==0$

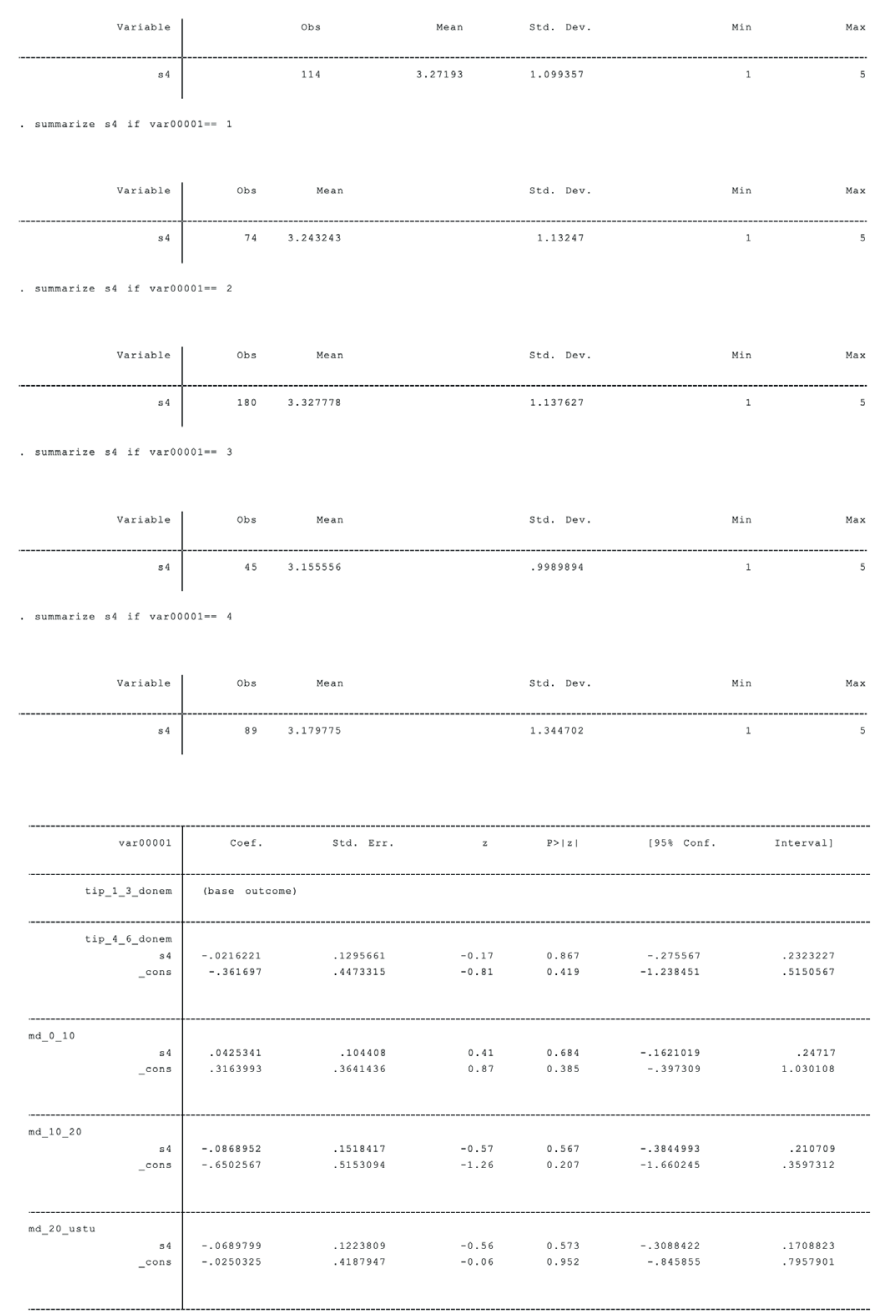

**** experience $\mathrm{s} 6{ }^{* *}$

. summarize $\mathrm{s} 6$ if $\operatorname{var} 00001==0$

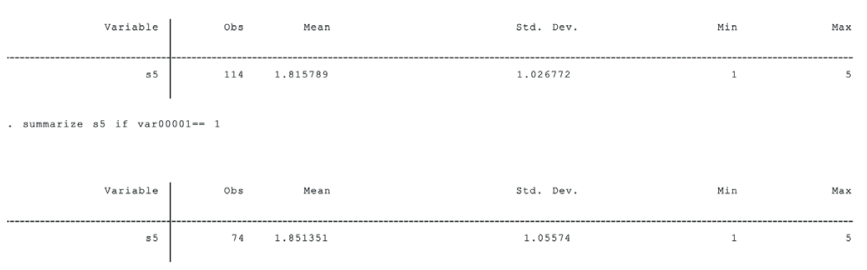

. surmarize s5 if var00001= $=$

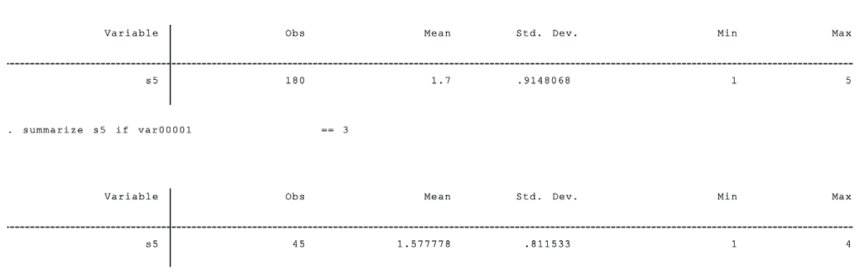

summarize s5 if var00001= 4

\begin{tabular}{c|ccccc} 
Variable & Dbs & Mean & std. Dev. & Min & Max \\
\hline 85 & 89 & 1.550562 & .9294578 & 1 & 5 \\
\hline & & & &
\end{tabular}




\begin{tabular}{|c|c|c|c|c|c|c|}
\hline vax 00001 & coos. & sta. Exr. & $=$ & $p>|z|$ & IDSt cont. & Interva 1 \\
\hline ttpp1-3_donem & (base outcone) & & & & & \\
\hline 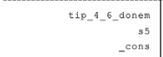 & 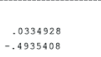 & $\begin{array}{l}.1245894 \\
.30657729\end{array}$ & $\begin{aligned} 0.223 \\
-1.62 \\
-1.62\end{aligned}$ & $\begin{array}{l}0.817 \\
0.1105\end{array}$ & $\begin{array}{l}-2.2999148 \\
-1.090967\end{array}$ & $\begin{array}{l}.31695004 \\
.12305856\end{array}$ \\
\hline - $\begin{array}{c}0.5 \\
-0000\end{array}$ & $\begin{array}{r}-.12190152 \\
.65725250\end{array}$ & $\begin{array}{l}.221212058 \\
.24515115\end{array}$ & $\begin{array}{c}-0.99 \\
2.122 \\
2.12\end{array}$ & $\begin{array}{l}0.323 \\
0.0007\end{array}$ & $\begin{array}{l}-3.35701212 \\
.106262519\end{array}$ & $\begin{array}{l}.1177138 \\
1.1118257\end{array}$ \\
\hline 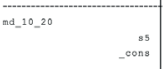 & $\begin{array}{r}-.272721253 \\
\hdashline-417306808\end{array}$ & $\begin{array}{l}.19779955 \\
.366870703\end{array}$ & 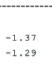 & $\begin{array}{l}0.171 \\
0.1977\end{array}$ & $\begin{array}{c}-.6564219 \\
-1,1,1920999\end{array}$ & $\begin{array}{l}.1161708 \\
.2460118\end{array}$ \\
\hline aA_Z20__astu & 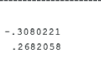 & 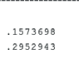 & $\begin{array}{l}-1.1696 \\
0.91\end{array}$ & $\begin{array}{l}0.050 \\
0.364\end{array}$ & 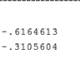 & $\begin{array}{r}.00001712 \\
.8669972\end{array}$ \\
\hline
\end{tabular}

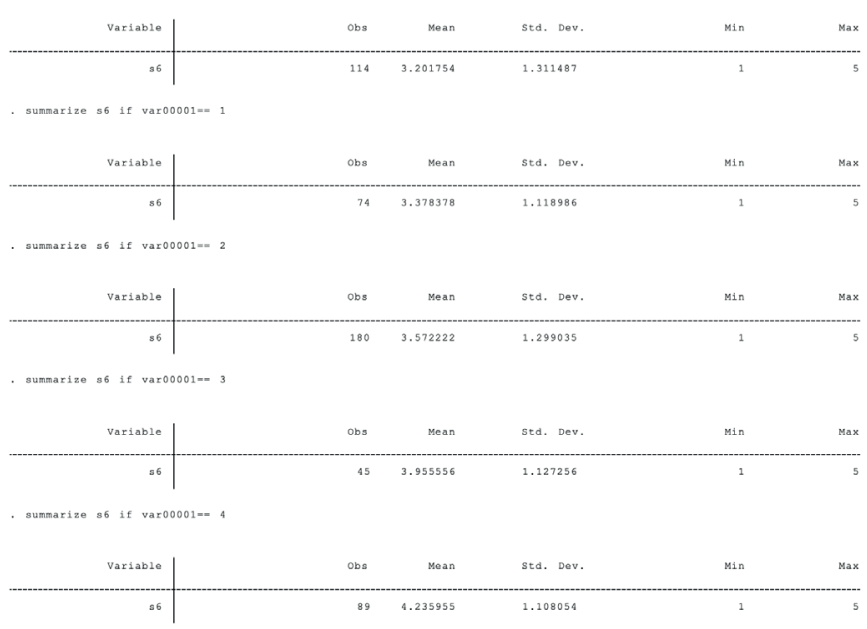

\section{**** experience $s 6^{* *}$} . summarize $\mathrm{s} 6$ if $\operatorname{var} 00001==0$

\begin{tabular}{|c|c|c|c|c|c|c|}
\hline varo0001 & coet. & sta. Exr. & $=$ & $p>121$ & 195\% cont. & Interval! \\
\hline tip_1_1_donen & (base outcome) & & & & & \\
\hline 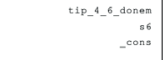 & $\begin{array}{r}.1015723 \\
-.766374\end{array}$ & $\begin{array}{l}.1136598 \\
.4049244\end{array}$ & $\begin{array}{c}0.89 \\
-1.89\end{array}$ & $\begin{array}{l}0.372 \\
0.058\end{array}$ & $\begin{array}{l}-.1211969 \\
-1.560011\end{array}$ & $\begin{array}{l}.3243414 \\
.0272632\end{array}$ \\
\hline $\begin{array}{r}36 \\
-\operatorname{cons} \\
\end{array}$ & $\begin{array}{r}.2194041 \\
-.2871184\end{array}$ & $\begin{array}{l}.0925215 \\
.3331799\end{array}$ & $\begin{array}{r}2.37 \\
-0.86\end{array}$ & $\begin{array}{l}0.018 \\
0.389\end{array}$ & $\begin{array}{r}.0380653 \\
-.9401371\end{array}$ & $\begin{array}{r}.400743 \\
.3659002\end{array}$ \\
\hline $\begin{array}{r}86 \\
\text { _cons }\end{array}$ & $\begin{array}{r}\quad 0893766 \\
-2.691621\end{array}$ & $\begin{array}{l}.1525655 \\
.6059022\end{array}$ & $\begin{array}{r}3.21 \\
-4.44\end{array}$ & $\begin{array}{l}0.001 \\
0.000\end{array}$ & $\begin{array}{r}.1903538 \\
-3.879168\end{array}$ & $\begin{array}{r}.7883999 \\
-1.500075\end{array}$ \\
\hline nd__20_ustu & $\begin{array}{r}.7480264 \\
-3.070794\end{array}$ & $\begin{array}{l}.1349959 \\
.551923\end{array}$ & $\begin{array}{r}5.54 \\
-5.56\end{array}$ & $\begin{array}{l}0.000 \\
0.000\end{array}$ & $\begin{array}{r}.4834399 \\
-4.152543\end{array}$ & $\begin{array}{r}1.012613 \\
-1.989045\end{array}$ \\
\hline
\end{tabular}

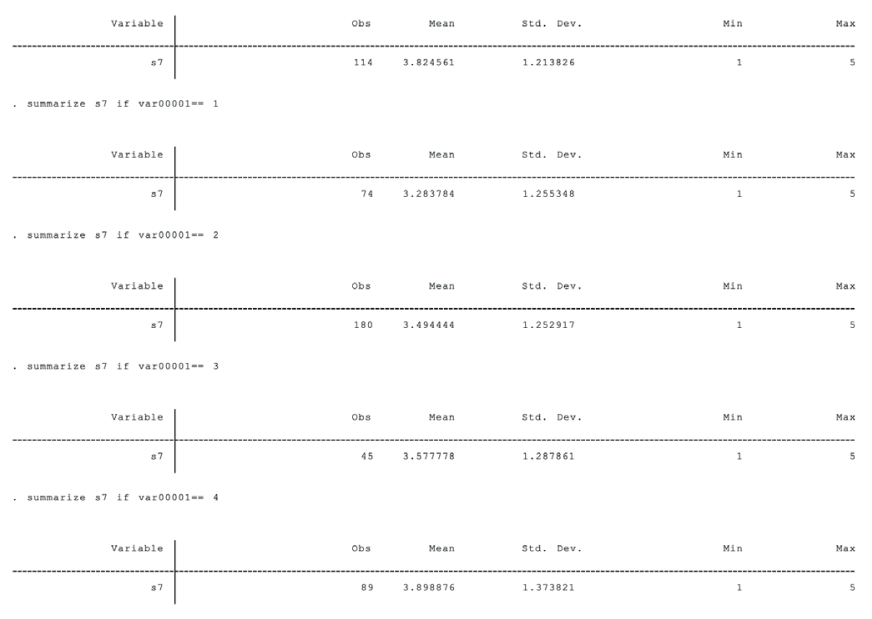

\begin{tabular}{|c|c|c|c|c|c|c|}
\hline varo0001 & coet. & std. Err. & $z$ & $p>|z|$ & 1951 cont. & Interval1 \\
\hline tip 1____donem & (base outcome) & & & & & \\
\hline $\begin{array}{r}\text { tip_-4 }{ }_{-}^{6} \text { _donem } \\
37 \\
\text { cons }\end{array}$ & $\begin{array}{r}. .3292995 \\
.741149\end{array}$ & $\begin{array}{l}.1174609 \\
.4410179\end{array}$ & $\begin{array}{r}-2.80 \\
1.68\end{array}$ & $\begin{array}{l}0.005 \\
0.093\end{array}$ & $\begin{array}{r}-.5595185 \\
-.1232303\end{array}$ & $\begin{array}{r}-.0990804 \\
1.605528\end{array}$ \\
\hline $\begin{array}{r}s ? \\
-\cos s\end{array}$ & $\begin{array}{l}-.208241 \\
1.219656\end{array}$ & $\begin{array}{l}.0967245 \\
.3783561\end{array}$ & $\begin{array}{r}-2.15 \\
3.22\end{array}$ & $\begin{array}{l}0.031 \\
0.001\end{array}$ & $\begin{array}{r}-.3978174 \\
.4780912\end{array}$ & $\begin{array}{r}-.0186645 \\
1.96122\end{array}$ \\
\hline $\begin{array}{r}57 \\
-\operatorname{cons}\end{array}$ & $\begin{array}{l}-.1583636 \\
-.3430206\end{array}$ & $\begin{array}{l}.1397762 \\
.5404419\end{array}$ & $\begin{array}{l}-1.13 \\
-0.63\end{array}$ & $\begin{array}{l}0.257 \\
0.526\end{array}$ & $\begin{array}{r}-.43232 \\
-1.402267\end{array}$ & $\begin{array}{l}.1155928 \\
.7162262\end{array}$ \\
\hline nd_20_ustu & $\begin{array}{r}.051992 \\
-.4483557\end{array}$ & $\begin{array}{r}.118546 \\
.4797815\end{array}$ & $\begin{array}{c}0.44 \\
-0.93\end{array}$ & $\begin{array}{l}0.661 \\
0.350\end{array}$ & $\begin{array}{r}-.1803539 \\
-1.39871\end{array}$ & $\begin{array}{l}.2843378 \\
.4919988 \\
.498\end{array}$ \\
\hline
\end{tabular}

*** experience $\mathrm{s} 8^{* * *}$

summarize $s 8$ if $\operatorname{var} 00001==0$

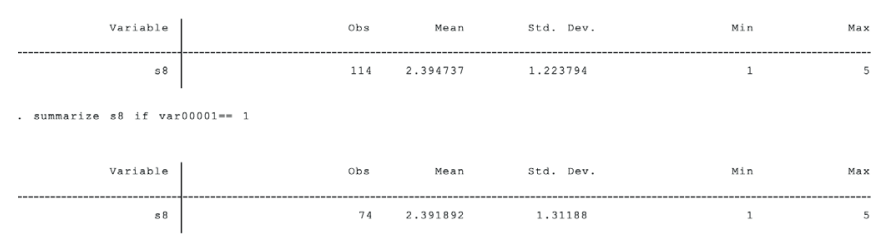

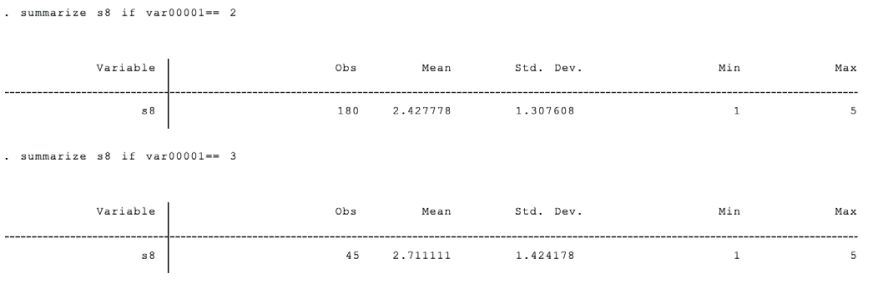

sumnarize s8 it varpooo1= 4

\begin{tabular}{c|ccccc} 
var1ab1e & obs & Mean & std. Dev. & Min & Max \\
\hline s8 & 89 & 2.550562 & 1.552207 & 1 & 5
\end{tabular} 


\begin{tabular}{|c|c|c|c|c|c|c|}
\hline va 000001 & coef. & std. $\mathbb{E}_{r r}$ & $=$ & $p>|z|$ & 1954 Conf. & Intervall \\
\hline tip_1 ${ }_{-}^{3}{ }^{-}$donem & (base outcome) & & & & & \\
\hline $\begin{array}{r}t 1 p_{-} 4-6 \text { - donem } \\
00 \\
\text { cons }\end{array}$ & $\begin{array}{r}-.0016237 \\
-.04282474\end{array}$ & $\begin{array}{r}.1127849 \\
.30843\end{array}$ & $\begin{array}{l}-0.01 \\
-1.39\end{array}$ & $\begin{array}{l}0.989 \\
0.165\end{array}$ & $\begin{array}{r}-.222679 \\
-1.032759\end{array}$ & $\begin{array}{l}.2194307 \\
.1762642\end{array}$ \\
\hline $\begin{array}{r}s \theta \\
-\operatorname{cons}\end{array}$ & $\begin{array}{r}.018712 \\
.4116396\end{array}$ & $\begin{array}{l}.0901566 \\
.2478684\end{array}$ & $\begin{array}{l}0.21 \\
1.66\end{array}$ & $\begin{array}{l}0.836 \\
0.097\end{array}$ & $\begin{array}{l}-.1579916 \\
-.0741735\end{array}$ & $\begin{array}{l}.1954157 \\
.8974527\end{array}$ \\
\hline md_10_20 & $\begin{array}{r}.1706357 \\
-1.364705\end{array}$ & $\begin{array}{l}.1295192 \\
.3798346\end{array}$ & $\begin{array}{r}1.33 \\
-3.59\end{array}$ & $\begin{array}{l}0.184 \\
0.000\end{array}$ & $\begin{array}{l}-.0812573 \\
-2.109167\end{array}$ & $\begin{array}{r}.4225286 \\
-.6202428\end{array}$ \\
\hline ma_zo_ustu & $\begin{array}{r}.0861822 \\
-.4605947\end{array}$ & $\begin{array}{l}.1051616 \\
.2967998\end{array}$ & $\begin{array}{r}0.82 \\
-1.55\end{array}$ & $\begin{array}{l}0.412 \\
0.121\end{array}$ & $\begin{array}{l}-.1199308 \\
-1.042312\end{array}$ & $\begin{array}{l}.2922951 \\
.1211221 \\
\end{array}$ \\
\hline
\end{tabular}

\begin{tabular}{r|rrrrr} 
Variable & obs & Mean & Std. Dev. & Min & Max \\
\hline 39 & 114 & 1.385965 & .6311777 & 1 & 4
\end{tabular}

. summarize 59 if var00001=- 1

\begin{tabular}{|c|c|c|c|c|c|}
\hline Variable & obs & Mean & std, Dev. & Min & $\operatorname{Max}$ \\
\hline$s 9$ & 74 & .594595 & .8426787 & 1 & 4 \\
\hline
\end{tabular}

. summarize 59 if var00001= $=2$

\begin{tabular}{r|rrrrr} 
Variable & obs & Mean & Std. Dev. & Min & Max \\
\hline$s 9$ & 180 & 1.327778 & .614813 & 1 & 5
\end{tabular}

\begin{tabular}{|c|c|c|c|c|c|}
\hline Variable & obs & Mean & sta. Dev. & Min & Max \\
\hline 59 & 45 & 1.24444 & .5289593 & 1 & 3 \\
\hline \multicolumn{6}{|c|}{ summarize $s 9$ if var00001= 4} \\
\hline variable & obs & Mean & std. Dev. & Min & Max \\
\hline 89 & 89 & 1.460674 & .8401323 & 1 & 5 \\
\hline
\end{tabular}

\section{*** experience $s 9^{* *}$ \\ . summarize $s 9$ if var00001 $==0$}

\begin{tabular}{|c|c|c|c|c|c|c|}
\hline var00001 & Coef. & std. BrI. & $=$ & $P>|z|$ & 1958 conf. & Interval] \\
\hline tip_1_-3_donem & (base outcome) & & & & & \\
\hline $\begin{array}{r}\text { tip_4_-6_donem } \\
39 \\
\text { cons }\end{array}$ & $\begin{array}{r}.3506958 \\
-.9516776\end{array}$ & $\begin{array}{l}.1942863 \\
.3256911\end{array}$ & $\begin{array}{r}1.81 \\
-2.92\end{array}$ & $\begin{array}{l}0.071 \\
0.003\end{array}$ & $\begin{array}{l}-.0300985 \\
-1.590021\end{array}$ & $\begin{array}{r}.73149 \\
-.3133347\end{array}$ \\
\hline $\begin{array}{r}59 \\
\text { cons }\end{array}$ & $\begin{array}{r}-.1396997 \\
.6461837\end{array}$ & $\begin{array}{l}.1842302 \\
.2778445\end{array}$ & $\begin{array}{r}-0.76 \\
2.33\end{array}$ & $\begin{array}{l}0.448 \\
0.020\end{array}$ & $\begin{array}{r}-.5007842 \\
.1016185\end{array}$ & $\begin{array}{l}.2213848 \\
1.190749\end{array}$ \\
\hline nd_ 10 - 20 & $\begin{array}{r}-.399525 \\
-.4065506\end{array}$ & $\begin{array}{l}.3192732 \\
.4428289\end{array}$ & $\begin{array}{l}-1.25 \\
-0.92\end{array}$ & $\begin{array}{l}0.211 \\
0.359\end{array}$ & $\begin{array}{l}-1.025289 \\
-1.274479\end{array}$ & $\begin{array}{r}.226239 \\
.4613782\end{array}$ \\
\hline $\begin{array}{r}\text { nd_20_ustu } \\
\begin{array}{r}s 9 \\
-\operatorname{cons}\end{array}\end{array}$ & $\begin{array}{l}.1468584 \\
-.456398\end{array}$ & $\begin{array}{l}.1980189 \\
.3155623\end{array}$ & $\begin{array}{r}0.74 \\
-1.45\end{array}$ & $\begin{array}{l}0.458 \\
0.148\end{array}$ & $\begin{array}{l}-.2412516 \\
-1.074889\end{array}$ & $\begin{array}{l}.5349684 \\
.1620927\end{array}$ \\
\hline
\end{tabular}

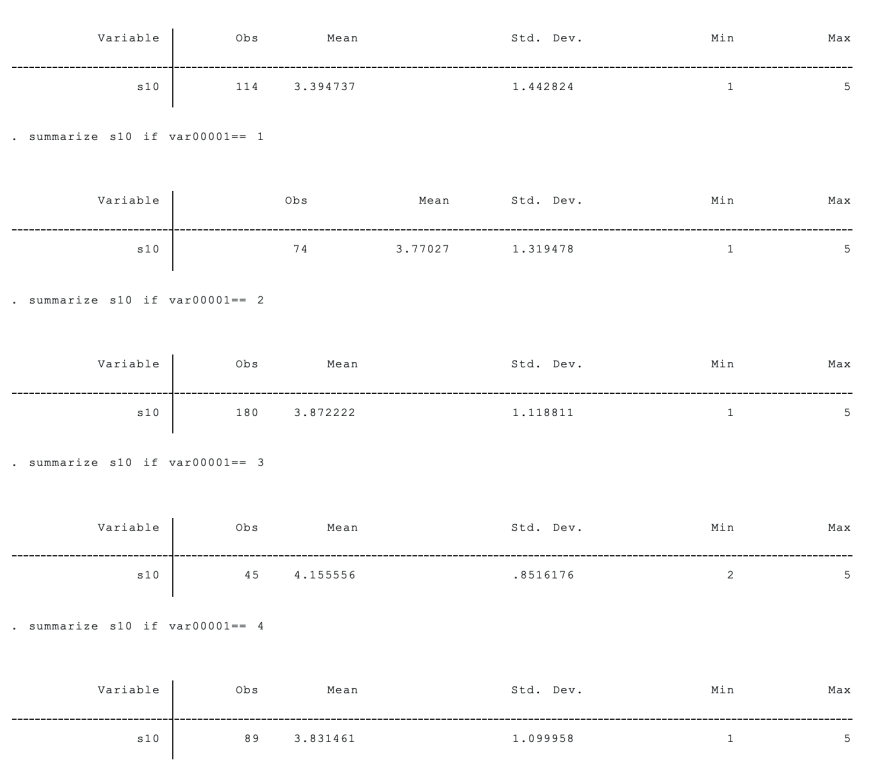

*** experience $\mathrm{s} 10^{* *}$

. summarize $\mathrm{s} 10$ if $\operatorname{var} 00001==0$

\begin{tabular}{|c|c|c|c|c|c|c|}
\hline $\operatorname{var} 00001$ & coef. & Std. Err. & $z$ & $P>|z|$ & [95\% conf. & Interval] \\
\hline tip______ & (base outcome) & & & & & \\
\hline \multicolumn{7}{|l|}{ tip_4_-6_donem } \\
\hline & .2284984 & .1191181 & 1.92 & 0.055 & -.0049688 & .4619655 \\
\hline _cons & -1.252262 & .459147 & -2.73 & 0.006 & -2.152174 & -.3523507 \\
\hline \multicolumn{7}{|l|}{ md_o_ 10} \\
\hline $\mathbf{s 1 0}$ & .3011576 & .0957498 & 3.15 & 0.002 & .1134914 & .4888239 \\
\hline _cons & -.6410716 & .3669797 & -1.75 & 0.081 & -1.360339 & .0781953 \\
\hline \multicolumn{7}{|l|}{ md__10_20 } \\
\hline$s 10$ & .5463032 & .1679655 & 3.25 & 0.001 & .2170969 & .8755096 \\
\hline _cons & -3.013181 & .6989685 & -4.31 & 0.000 & -4.383134 & -1.643228 \\
\hline \multicolumn{7}{|l|}{ md_20_ustu } \\
\hline $\mathrm{s} 10$ & .2713656 & .1143051 & 2.37 & 0.018 & .0473317 & .4953995 \\
\hline _cons & -1.230634 & .4430566 & -2.78 & 0.005 & -2.099009 & -.3622593 \\
\hline
\end{tabular}

\begin{tabular}{r|rrrrr} 
Variable & Obs & Mean & Std. Dev. & Min & Max \\
\hline s11 & 114 & 3.131579 & 1.258691 & 1 & 5
\end{tabular}

. summarize s11 if var00001== 1

\begin{tabular}{r|ccccr} 
Variable & Obs & Mean & Std. Dev. & Min & Max \\
\hline s11 & 74 & 3.067568 & 1.368241 & 1 & 5
\end{tabular}

- summarize s11 if $\operatorname{var00001==2}$

\begin{tabular}{r|rrrrr} 
Variable & Obs & Mean & Std. Dev. & Min & Max \\
\hline s11 & 180 & 2.622222 & 1.134308 & 1 & 5
\end{tabular}

- summarize $\mathrm{s} 11$ if $\operatorname{var} 00001==3$

\begin{tabular}{r|rrrrr} 
Variable & Obs & Mean & Std. Dev. & Min & Max \\
\hline s11 & 45 & 2.466667 & .8686458 & 1 & 4
\end{tabular}

. summarize s11 if var00001== 4

\begin{tabular}{r|ccccr} 
Variable & Obs & Mean & Std. Dev. & Min & Max \\
\hline sl1 & 89 & 2.606742 & 1.154221 & 1 & 5
\end{tabular}

*** experience $\mathrm{s} 11^{* *}$

summarize $\mathrm{s} 11$ if $\operatorname{var} 00001==0$ 


\begin{tabular}{|c|c|c|c|c|c|c|}
\hline var00001 & Coef. & Sta. Err. & $z$ & $P>|z|$ & [95\% Conf. & Interval] \\
\hline tip_1_3_donem & \multicolumn{6}{|c|}{ (base outcome) } \\
\hline tip_4_6_donem & & & & & & \\
\hline s11 & -.0451681 & .1253938 & -0.36 & 0.719 & -.2909355 & .2005992 \\
\hline _cons & -.29213 & .415583 & -0.70 & 0.482 & -1.106658 & .5223978 \\
\hline \multicolumn{7}{|l|}{ md_o_ 10} \\
\hline s11 & -.3598969 & .1026326 & -3.51 & 0.000 & -.561053 & -.1587408 \\
\hline _cons & 1.491786 & .324613 & 4.60 & 0.000 & .8555562 & 2.128016 \\
\hline \multicolumn{7}{|l|}{ md_10_20 } \\
\hline s11 & -.4748687 & .1533244 & -3.10 & 0.002 & -.7753789 & -.1743584 \\
\hline _cons & .3979689 & .4450049 & 0.89 & 0.371 & -.4742247 & 1.270162 \\
\hline \multicolumn{7}{|l|}{ md_20_ustu } \\
\hline s11 & -.3711372 & .1212295 & -3.06 & 0.002 & -.6087427 & -.1335318 \\
\hline _cons & .8168531 & .3728672 & 2.19 & 0.028 & .0860469 & 1.547659 \\
\hline
\end{tabular}

\begin{tabular}{|c|c|c|c|c|c|}
\hline variable & obs & Mean & std. Dev. & Min & Max \\
\hline $\mathbf{s 1 2}$ & 114 & 3.850877 & 1.074503 & 1 & 5 \\
\hline
\end{tabular}

. summarize $s 12$ if var00001== 1

\begin{tabular}{r|rrrrr} 
Variable & obs & Mean & Std. Dev. & Min & Max \\
\hline s12 & 74 & 4.121622 & 1.084798 & 1 & 5
\end{tabular}

. sumarize $s 12$ if var00001== 2

\begin{tabular}{r|rrrrr} 
Variable & Obs & Mean & Std. Dev. & Min & Max \\
\hline s12 & 180 & 4.072222 & 1.00296 & 1 & 5
\end{tabular}

\begin{tabular}{|c|c|c|c|c|c|}
\hline variable & obs & Mean & Std. Dev. & Min & Max \\
\hline $\mathbf{s 1 2}$ & 45 & .955556 & 1.1862 & 1 & 5 \\
\hline
\end{tabular}

. surmarize 512 if var00001= 4

\begin{tabular}{r|rrrrr} 
Variable & obs & Mean & Std. Dev. & Min & Max \\
\hline $\mathbf{s 1 2}$ & 89 & 4.191011 & .9519884 & 1 & 5
\end{tabular}

\begin{tabular}{|c|c|c|c|c|c|c|}
\hline $\operatorname{var} 00001$ & coef. & Std. Err. & $z$ & $p>|z|$ & 1958 conf. & Interva1] \\
\hline${ }^{t i P_{-}{ }_{-}{ }_{3}{ }_{-} \text {donem }}$ & (base outcome) & & & & & \\
\hline $\begin{array}{r}\text { tip_-4_6_donom } \\
\text { s12 } \\
- \text { cons }\end{array}$ & $\begin{array}{l}.2405287 \\
-1.39233\end{array}$ & $\begin{array}{l}.1442094 \\
.6004147\end{array}$ & $\begin{array}{r}1.67 \\
-2.32\end{array}$ & $\begin{array}{l}0.095 \\
0.020\end{array}$ & $\begin{array}{l}-.0421165 \\
-2.569121\end{array}$ & $\begin{array}{r}.5231739 \\
-.2155388\end{array}$ \\
\hline 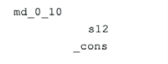 & $\begin{array}{r}.1917206 \\
-.3034645\end{array}$ & $\begin{array}{l}.1111341 \\
.4549168\end{array}$ & $\begin{array}{r}1.73 \\
-0.67\end{array}$ & $\begin{array}{l}0.085 \\
0.505\end{array}$ & $\begin{array}{l}-.0260983 \\
-1.195085\end{array}$ & $\begin{array}{l}.4095395 \\
.5881561\end{array}$ \\
\hline 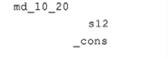 & $\begin{array}{r}.0858864 \\
-1.264834\end{array}$ & $\begin{array}{l}.1611796 \\
.6571346\end{array}$ & $\begin{array}{r}0.53 \\
-1.92\end{array}$ & $\begin{array}{l}0.594 \\
0.054\end{array}$ & $\begin{array}{l}-.2300199 \\
-2.552794\end{array}$ & $\begin{array}{r}.4017927 \\
.023126\end{array}$ \\
\hline $\begin{array}{r}\text { ma_20_ustu } \\
\text { s12 } \\
\text { _cons }\end{array}$ & $\begin{array}{r}.3139949 \\
-1.513143\end{array}$ & $\begin{array}{l}.1400075 \\
.5874146\end{array}$ & $\begin{array}{r}2.24 \\
-2.58\end{array}$ & $\begin{array}{l}0.025 \\
0.010\end{array}$ & $\begin{array}{r}.0395852 \\
-2.664455\end{array}$ & $\begin{array}{l}.5884046 \\
-.361832\end{array}$ \\
\hline
\end{tabular}

\begin{tabular}{r|rrrrr} 
Variable & obs & Mean & Std. Dev. & Min & Max \\
\hline s13 & 114 & 3.605263 & 1.156883 & 1 & 5
\end{tabular}

. sumnarize s13 if var00001== 1

\begin{tabular}{r|rrrrr} 
Variable & obs & Mean & Std. Dev. & Min & Max \\
\hline${ }^{113}$ & ${ }^{74}$ & 3.783784 & 1.138014 & 1 & 5
\end{tabular}

. summarize s13 if var00001= 2

\begin{tabular}{r|rrrrr} 
Variable & obs & Mean & Std. Dev. & Min & Max \\
\hline $\mathbf{s 1 3}$ & 180 & 3.722222 & 1.088567 & 1 & 5
\end{tabular}

. sumnarize s13 if var00001= 3

\begin{tabular}{c|ccccc} 
Variable & obs & Mean & Std. Dev. & Min & Max \\
\hline s13 & ${ }^{45}{ }^{3.711111}$ & 1.079188 & 1 & 5
\end{tabular}

summarize s13 if var00001=e 4

\begin{tabular}{r|rrrrr} 
Variable & obs & Mean & Std. Dev. & Min & Max \\
\hline 513 & 89 & ${ }^{4.011236}$ & 1.027955 & 1 & 5
\end{tabular}

\footnotetext{
*** experience $\mathrm{s} 13^{* *}$

. summarize $\mathrm{s} 13$ if $\operatorname{var} 00001==0$
}

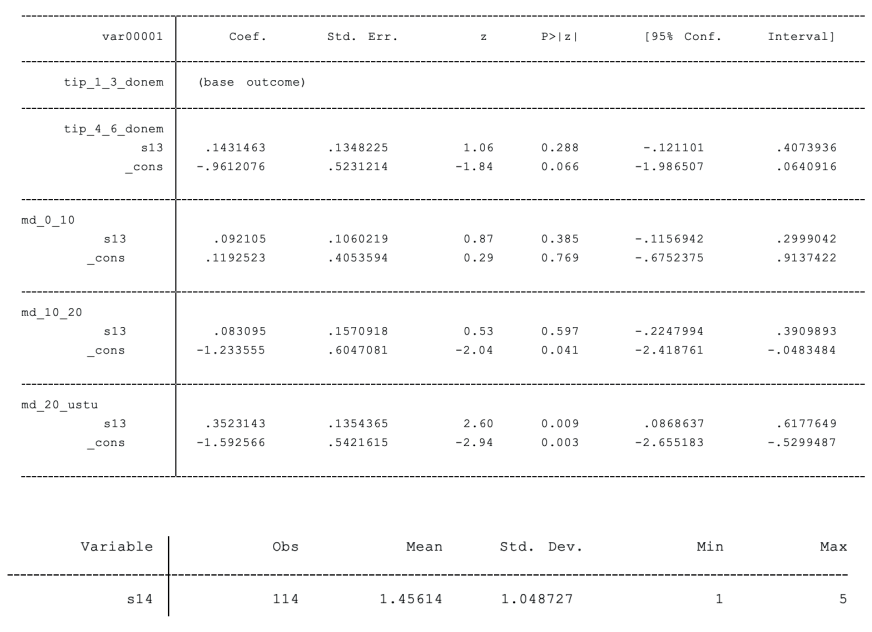

. summarize $s 14$ if var00001== 1

\begin{tabular}{|c|c|c|c|c|c|}
\hline Variable & obs & Mean & Std. Dev. & Min & $\operatorname{Max}$ \\
\hline$s 14$ & 74 & 1.459459 & .939093 & 1 & 5 \\
\hline
\end{tabular}

. summarize s14 if var00001== 2

\begin{tabular}{c|cccrr} 
Variable & obs & Mean & Std. Dev. & Min & Max \\
\hline$-\mathbf{y}^{14}$ & 180 & 1.383333 & .8274279 & 1 & 5
\end{tabular}

. summarize s14 if var00001== 3

\begin{tabular}{r|cccrr} 
Variable & obs & Mean & Std. Dev. & Min & Max \\
\hline s14 & 45 & 1.4 & 1.074498 & 1 & 5
\end{tabular}

. summarize s14 if var00001= 4

\begin{tabular}{r|rrrrr} 
Variable & obs & Mean & Std. Dev. & Min & Max \\
\hline s14 & 89 & 1.853933 & 1.434562 & 1 & 5
\end{tabular}




\begin{tabular}{|c|c|c|c|c|c|c|}
\hline var00001 & Coef. & Std. Err. & $z$ & $p>121$ & [95* Conf. & Interval] \\
\hline tip_1_3donem & (base outcome) & & & & & \\
\hline $\begin{array}{r}\text { tip_4_6_donem } \\
914 \\
\text { cons }\end{array}$ & $\begin{array}{r}.0033056 \\
-.0369523\end{array}$ & $\begin{array}{l}.1489161 \\
.2635069\end{array}$ & $\begin{array}{r}0.02 \\
-1.66\end{array}$ & $\begin{array}{l}0.982 \\
0.097\end{array}$ & $\begin{array}{l}-.2885645 \\
-.9534164\end{array}$ & $\begin{array}{l}.2951757 \\
.0795118\end{array}$ \\
\hline $\begin{array}{r}\text { md____10 } \\
\quad \begin{array}{r}\text { s14 } \\
\text {-cons }\end{array}\end{array}$ & $\begin{array}{r}-.0802753 \\
.5705313\end{array}$ & $\begin{array}{l}.1247742 \\
.2143241\end{array}$ & $\begin{array}{r}-0.64 \\
2.66\end{array}$ & $\begin{array}{l}0.520 \\
0.000\end{array}$ & $\begin{array}{r}-.3248283 \\
.1505637\end{array}$ & $\begin{array}{l}.1642777 \\
.9906988\end{array}$ \\
\hline $\begin{array}{r}\text { md_ }_{-}{ }^{-}{ }_{-2}^{20} \\
\text { s14 } \\
\text { - cons }\end{array}$ & $\begin{array}{l}-.0604352 \\
-.9032726\end{array}$ & $\begin{array}{r}.1859862 \\
.316543\end{array}$ & $\begin{array}{l}-0.32 \\
-2.66\end{array}$ & $\begin{array}{l}0.745 \\
0.008\end{array}$ & $\begin{array}{l}-.4249613 \\
-1.463685\end{array}$ & $\begin{array}{r}.304091 \\
-.2228597\end{array}$ \\
\hline $\begin{array}{r}\text { md_20_ustu } \\
\text { s14 } \\
\text {-cons }\end{array}$ & $\begin{array}{r}.2812621 \\
-.71071639\end{array}$ & $\begin{array}{l}.121924 \\
.2437866\end{array}$ & $\begin{array}{r}2.31 \\
-2.90\end{array}$ & $\begin{array}{l}0.021 \\
0.004\end{array}$ & $\begin{array}{r}.0422954 \\
-1.184977\end{array}$ & $\begin{array}{r}.5202287 \\
-.2293509\end{array}$ \\
\hline
\end{tabular}

\begin{tabular}{r|rrrrr} 
Variable & obs & Mean & Std. Dev. & Min & Max \\
\hline s15 & 114 & 1.815789 & 1.347339 & 1 & 5
\end{tabular}

summarize s15 if var00001== 1

\begin{tabular}{r|rrrrr} 
Variable & obs & Mean & Std. Dev. & Min & Max \\
\hline $\mathbf{s 1 5}$ & 74 & 1.837838 & 1.216632 & 1 & 5
\end{tabular}

summarize s15 if var00001==2

\begin{tabular}{r|rrrrr} 
Variable & obs & Mean & Std. Dev. & Min & Max \\
\hline s15 & 180 & 2.011111 & 1.353617 & 1 & 5
\end{tabular}

. summarize s15 if var00001== 3

\begin{tabular}{r|rrrrr} 
Variable & obs & Mean & Std. Dev. & Min & Max \\
\hline s15 & 45 & 1.844444 & 1.380967 & 1 & 5
\end{tabular}

. summarize s15 if var00001==4

\begin{tabular}{r|rrrrr} 
Variable & obs & Mean & Std. Dev. & Min & Max \\
\hline $\mathbf{y} 15$ & 89 & 2.550562 & 1.644628 & 1 & 5
\end{tabular}

*** experience $\mathrm{s} 15^{* *}$

summarize $\mathrm{s} 15$ if var00001= $=0$

\begin{tabular}{|c|c|c|c|c|c|c|}
\hline var00001 & Coef. & Std. Err. & $z$ & $P>|z|$ & 1958 conf. & Interval] \\
\hline tip_1_3_donem & \multicolumn{6}{|l|}{ (base outcome) } \\
\hline \multicolumn{7}{|l|}{ tip_4_6_donem } \\
\hline & .0133331 & .1159519 & 0.11 & 0.908 & -.2139285 & .2405947 \\
\hline _cons & -.4564899 & .259355 & -1.76 & 0.078 & -.9648164 & .0518367 \\
\hline \multicolumn{7}{|l|}{ md_0_10 } \\
\hline s15 & .1089105 & .0906923 & 1.20 & 0.230 & -.0688432 & .2866641 \\
\hline _cons & .2486791 & .2087519 & 1.19 & 0.234 & -.1604671 & .6578253 \\
\hline \multicolumn{7}{|l|}{ md_10_20 } \\
\hline & .0172686 & .1362358 & 0.13 & 0.899 & -.2497487 & .2842859 \\
\hline _cons & -.9611383 & .3058963 & -3.14 & 0.002 & -1.560684 & -.3615926 \\
\hline \multicolumn{7}{|l|}{ md_20_ustu } \\
\hline s15 & .3466637 & .0996319 & 3.48 & 0.001 & .1513888 & .5419385 \\
\hline _cons & -.9951376 & .2590864 & -3.84 & 0.000 & -1.502938 & -.4873377 \\
\hline
\end{tabular}

